\title{
Seasonal Change on Titan Observed with the Hubble Space Telescope WFPC-2
}

\author{
Ralph D. Lorenz, Mark T. Lemmon, and Peter H. Smith \\ Lunar and Planetary Laboratory, University of Arizona, Tucson, Arizona 85721-0092 \\ and \\ G. W. Lockwood \\ Lowell Observatory, 1400 West Mars Hill Road, Flagstaff, Arizona 86001-4470
}

Received November 9, 1998; revised June 8, 1999

\begin{abstract}
Recent observations with the Wide-Field Planetary Camera (WFPC-2) on the Hubble Space Telescope (HST) show an unexpectedly rapid change in the atmospheric albedo contrast between the north and south hemispheres. In 1994 at blue wavelengths, the north was around $15 \%$ brighter than the south, and was expected to fall to about $12 \%$ in 1997 , but has dropped to only $6 \%$ brighter. At some other wavelengths, the contrast has reversed, which was not expected until 2002. The interhemispheric contrast has a time dependence that varies with wavelength; contrast changes in blue lag behind changes in violet and yellow/red. The rapid change and the phase variation with wavelength are consistent with ground-based photometry. A physical model of the transport of high-altitude dark haze by meridional winds is a better description of Titan's behavior than the simple sinusoidal models used to date. Investigation with a radiative transfer model indicates that haze number density changes above $160-\mathrm{km}$ altitude are compatible with the observed hemispheric albedo difference, and require particles $>0.1 \mu \mathrm{m}$ in radius. (c) 1999 A cademic Press
\end{abstract}

Key Words: Titan; atmospheres, dynamics; image processing; photometry; radiative transfer.

\section{INTRODUCTION}

Titan's thick hazy atmosphere not only obscured the surface from Voyager's cameras (Smith et al. 1981), but was itself deceptively bland in appearance, with the most notable feature being a sharp albedo difference between the north and south hemispheres (Sromovsky et al. 1981, hereafter S81). This so-called north-south asymmetry (NSA) has a peak magnitude when the Sun crosses Titan's equator every 14.5 years, or every 0.5 Titan year. This occurred in 1980 (when Voyager 1 arrived, in northern spring) and the asymmetry was expected to fall to zero after 7 years (i.e., in 1987). Early Hubble Space Telescope (HST) observations showed that the asymmetry had indeed reversed (Caldwell et al. 1992) between 1981 and 1990.
Because Titan was observed with good spatial resolution only close to equinox (by Voyagers 1 and 2 in 1980/1981, and HST/ WFPC-2 in 1994/1995), there was only information on the extrema of the NSA cycle. It was assumed that the variation was sinusoidal (Smith et al. 1981, 1982; S81). After the asymmetry peaked again (in the opposite sense, with north $15 \%$ brighter at blue wavelengths) in 1994/1995, it was predicted to fall to zero in 2002 (Lorenz et al. 1997, hereafter referred to as L97).

In this paper, we report on analyses of new images obtained in November 1997, two years after equinox. Using a new, more accurate data reduction, we compare these with images obtained in 1994 and 1995 with the same instrument and filters. We find that seasonal change on Titan appears to occur faster than was expected. We introduce a simple physical model for these changes, invoking the latitudinal transport of haze particles as suggested by Hutzell et al. (1996) and more recently elaborately modeled by Tokano et al. (1999), and then attempt to quantify the number density, optical properties, and altitude of the haze responsible using detailed radiative transfer models.

\section{OBSERVATIONS AND DATA REDUCTION}

\subsection{Qualitative Data Analysis: Titan's Appearance to HST}

Titan's appearance at equinox is well known at visible wavelengths from the Voyager data, which show a limb-darkened disk, with a difference in albedo between hemispheres. This difference in hemispheres is strongest in the blue and green and weaker at violet and red wavelengths. Additionally, at high northern latitudes, a dark polar collar or hood is seen. It has been hypothesized (Yung 1987) that this latter feature is due to the condensation of photochemical products that accumulate in the cold winter period when these latitudes are in shadow.

With HST, Titan appears broadly the same close to equinox, although HST's spatial resolution of about $300 \mathrm{~km}$ is far poorer 


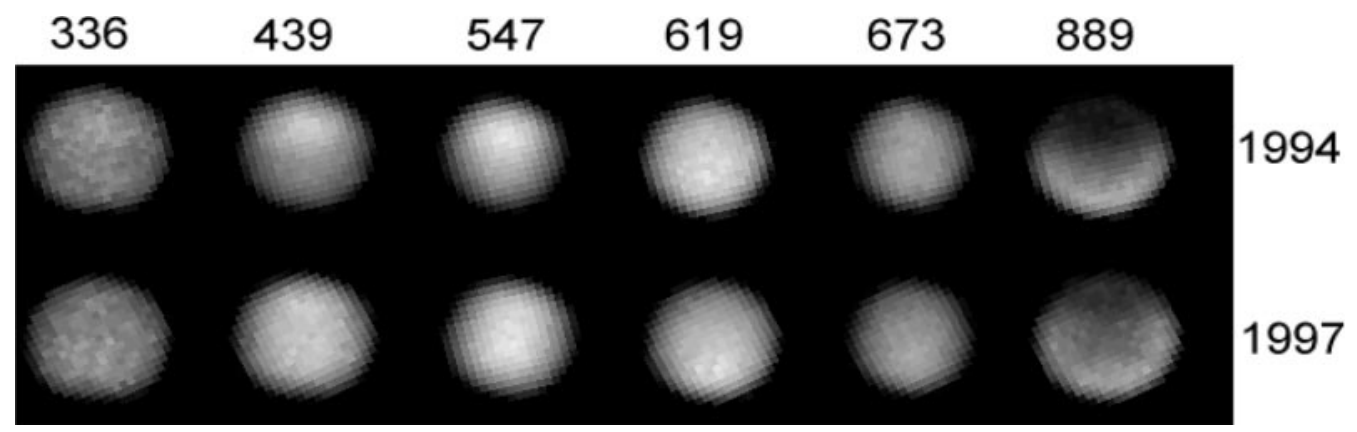

FIG. 1. HST Images of Titan in 1994 (top) and 1997 (bottom). From left to right the filters are F336W, F439W, F547M, FQCH4N-B (619 nm), F673N, and FQCH4N-D $(889 \mathrm{~nm})$. North is up, and all images are scaled to have the same maximum brightness. It can be seen that the strong asymmetry at 439 and $547 \mathrm{~nm}$ has virtually disappeared. The north at $619 \mathrm{~nm}$ has darkened somewhat, and the brightness at $889 \mathrm{~nm}$ has moved northward.

than the Voyagers'. The wavelength coverage of WFPC-2 is wider, notably permitting the surface to be imaged at near-IR wavelengths (Smith et al. 1996). The filters we use in this study are F336W, F439M, F547W, F588N, and F673N continuum filters, with $\mathrm{N}, \mathrm{M}$, and $\mathrm{W}$ denoting narrow, medium, and wide filters, and the narrow methane band filters FQCH4N-B and FQCH4N-D at 619 and $889 \mathrm{~nm}$ (Burrows 1994).

Titan as seen by HST in 1994 and 1997 is shown in Fig. 1. Considering the 1994 images first, limb darkening increases from violet through red wavelengths; this has the effect of making the disk look somewhat smaller at longer wavelengths, as seen in the figure (each $300-\mathrm{km}$ pixel is about one-tenth of a Titan radius). There is also an optical radius effect [see Toon et al. (1992) and discussion later], but this is not significant at this scale. The 889-nm methane band image has a rather different appearance (see Smith et al. 1996 and L97 for discussion): here there is limb brightening since the deep atmosphere is black due to methane absorption (with an absorption optical depth of about 100), and the thin haze above the bulk of the atmosphere is bright. Since the haze at these levels is optically thin, the longer pathlength through it leads to a bright limb.

The difference in albedo between the two hemispheres (the NSA) is apparent, with the difference strongest in green and blue, as in the Voyager data, two seasons before. The NSA is reversed in the methane band, due to bright haze being more abundant or reflective in the south. In 1997, the picture is broadly the same, except that the NSA appears to have reduced somewhat. The "smile" of the methane band image also seems to have thinned and widened (the brightness is creeping northward along the limb).

In 1997 (and, to a lesser extent, in 1995 images not shown), there is some faint indication of a "flat bottom" of Titan at 336 and $439 \mathrm{~nm}$ (see bottom left of Fig. 1). This is probably the dark "polar hood" observed in the northern hemisphere by Voyagers 1 and 2 (Smith et al. 1981, 1982). The hood in the north was observed to have disappeared by 1990 (Caldwell et al. 1992), as would be expected by a seasonal model. As we are now moving into southern spring, the south polar regions that have been shadowed are coming into view, and although the HST resolu- tion is poor, the polar hood seems to be present. In the next few years, our viewing geometry will continue to improve with the southward migration of the sub-Earth point. At the same time, however, the albedo contrast of the hood will decay as these regions accumulate more sunlight.

\subsection{Quantitative Image Analysis}

The images underwent standard STScI pipeline processing. The regions of the images containing Titan's disk were extracted and each disk was fit by a model Titan, comprising two model "hemispheres": the brightness for each pixel is given by $I_{0} \mu^{k} \mu_{0}^{k-1}$, with $\mu$ and $\mu_{0}$ the cosine of the zenith angle and the solar zenith angle, respectively. $I_{0}$ is the normal reflectivity, and $k$ the Minnaert coefficient. The principal parameter we shall be discussing in this paper is the ratio in $I_{0}$ values for the two hemispheres. Where we use the word contrast, this implies the quantity $I_{0}$ (bright) $/ I_{0}(\operatorname{dim})-1$.

The resultant model image was convolved with a synthetic point spread function (PSF) and the convolved image compared with the observed one. The model and comparison are made with $3 x$ oversampled images since the model brightness changes rapidly at the edge of the disk. The parameters are adjusted until the root mean squared (rms) difference between the model and observed images is minimized.

This analysis procedure improves on that in L97 [in turn derived from Sromovsky et al. (1981)] in several respects.

1. The parameter fits were generated automatically, using the downhill simplex method (implemented by the Interactive Data Language procedure AMOEBA), whereas in L97 the "optimization" was performed by hand, varying one parameter at a time. The new procedure provides significantly better fits, since the simplex method finds local minima in the cost function (here the mean square error of the fit) by systematically varying all parameters, whereas the manual procedure found them in a more heuristic fashion. Further, patience limitations in the manual fit limited the parameter variations to increments of one part in 1000 or higher: the automated procedure varies the stepsize down to one part in $10^{5}$. 
2. While L97 used midpoints between maximum-slope edges of the images to determine the centers, the new procedure iteratively finds the best center, taking into account in the process any phase angle effects.

3. The $\mu$ and $\mu_{0}$ values for each hemisphere must be computed on the basis of a radius. L97 used fixed values for the radius, specifically those of Toon et al. (1992), whereas the radius was a free parameter in the new determinations. Further, the north and south hemispheres had separate radii in the new fits.

4. L97 reported only one determination of north/south albedo ratio per image, with an estimated uncertainty. The new method, with many measurements, allows a better visibility of the confidence of each NSA determination and a more reliable determination of the errors: this aspect of the analysis is discussed in the following section.

\subsection{Results}

We have devoted particular attention in this work to understanding the uncertainty of our measurements of the NSA, since some of the changes we observe are subtle and surprising. We found that the RMS error in the fits was typically $3 \%$, fractionally better than achieved by hand in L97. The best fits $(\sim 1.5 \%$ RMS error) were obtained in the F588N filters, with the noisier F336W image reaching about $4 \%$. The 889 -nm images are not as well described by the Minnaert model, but nevertheless errors were around $6 \%$.

Repeated fits with different starting points yielded significant scatter in results. A Monte-Carlo approach was therefore tried, taking 20 optimized fits and taking either a mean or the fit with the lowest error, but this failed to produce significantly better results, the effectiveness being judged by comparing two nominally identical HST images (e.g., our two exposures in 1997 using the F439W filter; these should differ only by Poisson noise). For quantitative comparisons between the years, we therefore use the following method. A set (here, six) of converged fits was obtained, each fit with the ratio of $I_{0}$ values for the two hemispheres (i.e., the NSA) held to a single fixed value. This constrained NSA value was varied across the expected "true" value, typically in steps of 0.005 across a range of 0.1 , making some 120 fits per image. A plot of the errors against the NSA shows a clear minimum, and the location of the minimum is stable between nominally identical images. The NSA values we report in the present paper correspond to the minimum of a quadratic fit to the error. We found in our initial analysis that without several trial fits for each NSA value, the error envelope would not be well defined.

The uncertainties we report in the NSA values correspond to the width of the minimum, defined by one-third of the width at a threshold set at the minimum value of the fitted quadratic plus three times the standard deviation of the quadratic fit. Performing more image fits could presumably define the error curve rather better and hence slightly reduce the uncertainty. However, this would be expensive computationally, and is probably unjustified given that the two-hemisphere model is not a complete descrip- tion of the real Titan so some systematic (rather than statistical) uncertainty would remain.

Our results are shown in Fig. 2. Where we have more than one image in a given filter in a given year, the values generated by this method are always within 0.01 of each other, suggesting our method is robust. This small scatter is consistent with our uncertainty estimates (which describe the overall absolute uncertainty of our estimated NSA; the relative uncertainty may in fact be somewhat better than our error bars show).

\subsection{Discussion and Supporting Data}

The results and error bars from this new data reduction overlap with the earlier analyses of 1994 and 1995 data in L97, although they are generally fractionally smaller (e.g., the 1994 $\mathrm{NSA}=1.15$ at $547 \mathrm{~nm}$, compared with 1.18 in L97). This confirms the suspicion offered in L97 that the NSA in 1994/1995 (northern spring equinox) was rather weaker than the NSA at the corresponding Voyager epoch (1980/1981), where the bright/ dark hemisphere ratio was 1.25 . This difference is remarkable, but not altogether surprising. Titan's substantial orbital eccentricity modulates the insolation: Tokano et al. (1999) have recently determined that this eccentricity effect causes the peak stratospheric summer temperature over the south pole to be $10 \mathrm{~K}$ warmer than the peak summer temperature in the north. This temperature difference may affect condensation processes on the haze as well as the circulation that blows the haze around. It may be that Titan's haze follows an asymmetric seasonal cycle, in much the same way as water does on Mars with the consequence that the martian polar caps are different in size and composition from each other.

Sinusoidal fits to the NSA-versus-time data for the 19801995 period (L97) predicted that the blue contrast should be about $12 \%$ in 1997. However, we see (Fig. 2) that the asymmetry has dropped sharply, to only $6 \%$, and may be expected to fall to zero within the next 2 years. Evidently a smooth sinusoidal variation passing through the extrema of the cycle observed in $1980 / 1981$ and 1994/1995 is an inappropriate description of the NSA behavior.

The asymmetry's complex dependence on wavelength is well known (Tomasko and Smith, 1982; S81; L97; Caldwell et al. 1992). Given the previous assumption of a sinusoidal variation (with time), the expectation was that the asymmetry-versuswavelength curve would flip about the NSA $=1$ line, like the projection of a skip rope. We now observe (Fig. 2), however, that the asymmetry does not vary in phase at all wavelengths.

Particularly notable are the complete decay of the asymmetry at $336 \mathrm{~nm}$ and its reversal at $673 \mathrm{~nm}$; an in-phase sinusoidal variation of the NSA would not predict these changes until 2002. The implication is that changes at violet and red wavelengths lead those in blue by 1-2 years. The difference in behavior between 619 and $673 \mathrm{~nm}$ is particularly interesting, presumably due to the methane absorption at $619 \mathrm{~nm}$.

The fact that the changes in NSA in the 619- and 889-nm images are not in phase is also interesting. The large decrease 


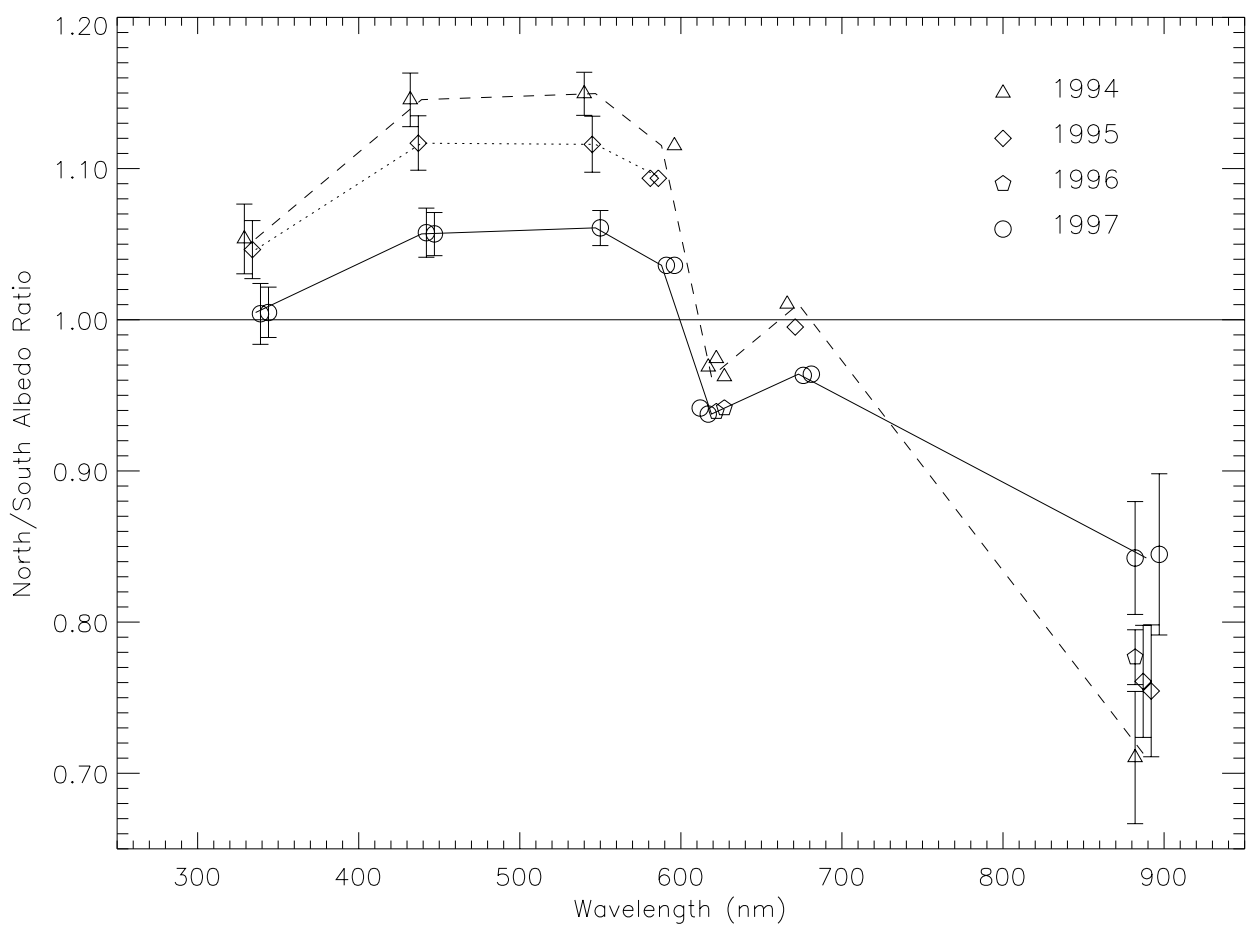

FIG . 2. Variation of the north/south albedo ratio with wavelength and time. Triangles connected with a dashed line denote 1994 observations, diamonds with dotted line 1995, and circles with a solid line 1997. Pentagons are methane band images from 1996. Uncertainties are indicated for several data points (omitted where the points are crowded.) In the methane bands at 619 and $889 \mathrm{~nm}$, the asymmetry is reversed in sense. While it has decreased in magnitude at 889 and $336-588 \mathrm{~nm}$, the asymmetry has intensified somewhat at $619 \mathrm{~nm}$ and reversed at $673 \mathrm{~nm}$. Symbols are displaced $\pm 10 \mathrm{~nm}$ to minimize crowding.

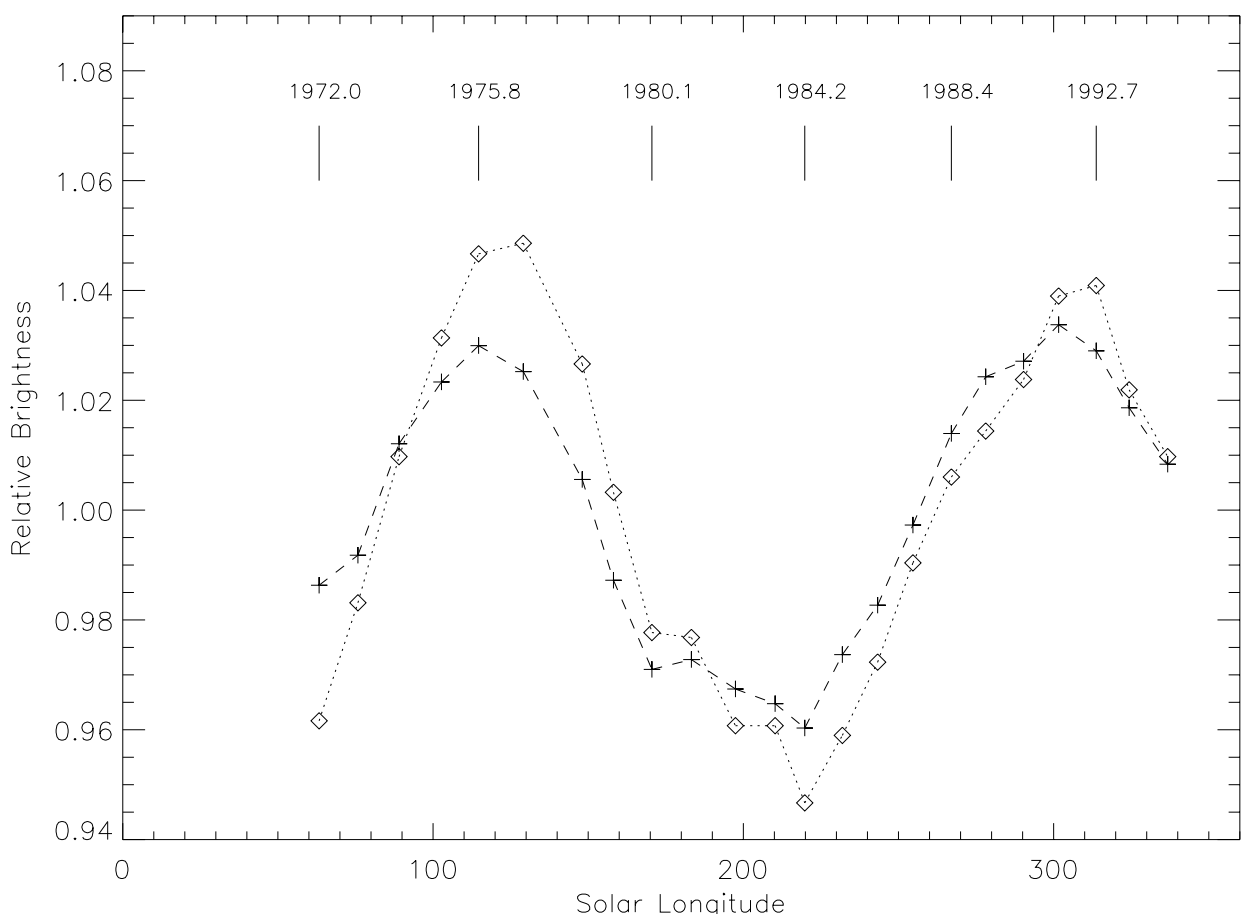

FIG . 3. Disk-integrated blue (diamonds with dotted line) and yellow (crosses with dashed line) photometry obtained at Lowell observatory; dates are shown at the top. The advanced phase of the yellow data is apparent: both the peaks and the center crossings occur about 0.5 year before the corresponding events in blue. Note that the solar longitude is the astronomically conventional solar longitude of Saturn; Tokano et al. (1999) use the climatological convention of $L_{\mathrm{s}}$, the saturnocentric longitude of the Sun. 
in NSA at $889 \mathrm{~nm}$ (the filter probing the higher altitude) suggests that the changes on Titan are occurring primarily at high altitudes.

Although little difference was observed between blue images taken by Voyager 1 encounter and by Voyager 2, 9 months later (Smith et al. 1982), there are indications (Table I of L97) that the contrast dropped by $4 \%$ at violet and green wavelengths over that interval, consistent with the trends we see in our HST data. The reduced errors in our present analysis of our 1994 and 1995 HST images also allow the detection of significant change between those years (Fig. 2).

A consistent picture emerges, then, of the blue lagging behind both longer and shorter wavelengths. There is some supporting evidence from ground-based photometry for this previously unsuspected wavelength-dependent phase of the NSA variation. Titan exhibits a change in disk-integrated albedo over time, which has been monitored since 1971 by Lockwood and Thompson (1979; Lockwood et al. 1986a,b). The albedo varies approximately as a sinusoid, with an amplitude of about $4 \%$ and a period of half a Titanian year. As shown in Fig. 3, the yellow cycle appears slightly phase-advanced with respect to the blue. Quantitatively, a best-fit albedo of the form $a_{0}+a_{1} \sin \left(2 \pi\left(t-t_{0}\right) / 14.75\right)$, with $t_{0}$ indicating the phase, yields amplitudes $\left(a_{1}\right)$ of 0.028 for yellow and 0.040 for blue, with $t_{0}$ values of 1987.40 and 1988.0, each with rms errors of 0.01 . Thus the yellow is therefore around 0.6 year ahead of the blue, consistent with our imaging observation of the NSA above.

We do not understand why the yellow/red contrast changes should lead those in the blue. The apparent lead of violet over blue is easier to understand, in that these short wavelengths sample only the uppermost atmosphere (see below). Tokano et al. (1999) notes that haze transport by seasonally driven winds leads to a change in haze number density at higher altitudes first; thus the NSA at violet wavelengths should be quickest to respond, as observed.

\subsection{Additional Imaging Results}

Our fitting procedure yields two additional parameters: a model radius and a limb-darkening coefficient. The Minnaert coefficient $k$ shown in Fig. 4 shows a pattern consistent with L97, although absolute values are 0.05-0.1 smaller. This offset is in part due to the different data reduction techniques [L97 used raw images, and corrected $k$ by an empirical 0.06 to allow for PSF effects, while the present analysis treats the PSF explicitly]. An additional reason for the difference is that in L97 the radius was forced to be equal to the Toon et al. (1992) values, while in the present analysis radius is a free parameter. The results here were obtained by a procedure similar to those for the NSA - holding both hemispheres to the same variable $k$ value and selecting the value that yielded the minimum error.

A new result in Fig. 4 is that the limb-darkening coefficient is smaller at $619 \mathrm{~nm}$ than at either 588 or $673 \mathrm{~nm}$. This is almost

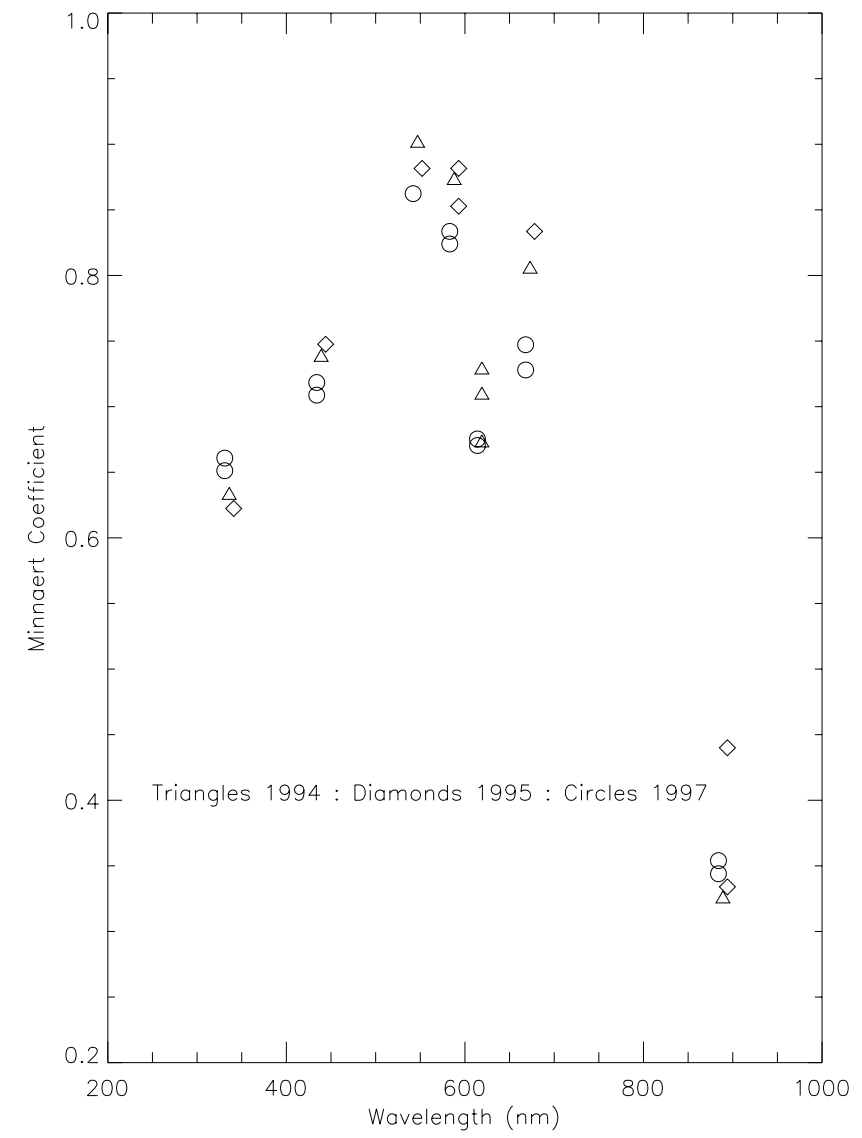

FIG . 4. Minnaert coefficient as a function of wavelength. The increasing limb darkening from 336 to $588 \mathrm{~nm}$ and trend toward limb brightening at $889 \mathrm{~nm}$ are as noted in L97, although note the strong dip at $619 \mathrm{~nm}$. Some interannual variability is apparent at $673 \mathrm{~nm}$.

certainly due to methane absorption at $619 \mathrm{~nm}$ causing the disk center to be less bright relative to the limb than at the adjacent wavelengths. There is also evidence of a temporal change in the limb-darkening coefficient: above $550 \mathrm{~nm}$ between 1994/1995 and 1997 it drops by about 0.05 .

A separate data reduction allowing both radius and $k$ to vary for each hemisphere indicated a larger radius for the northern hemisphere above $550 \mathrm{~nm}$ and a higher Minnaert coefficient. However, the scatter in these data was large (since a large radiuslarge $k$ and a small radius-small $k$ may give equally good fits) so we present results for fits using $k$ constrained to be the same for both hemispheres.

The values for radius are shown in Fig. 5 and compared with previous measurements [although note that not all radii are equal; we show here the best-fit Minnaert model radius, while the Karkoschka and Lorenz (1997) results are for a grazing optical depth of 0.028; the analytic expression from Toon et al. (1992) is a fit to a variety of data]. This caveat aside, the general agreement is good, although the data fall below the line at 619 and $673 \mathrm{~nm}$ : Titan's upper atmosphere seems more transparent at these wavelengths than models indicate. The Minnaert fit is 
poor for the limb-brightened southern hemisphere at $889 \mathrm{~nm}$; we do not report those radius values.

Figure 6 shows HST photometry of Titan. We measured Titan's total brightness (we summed pixels within a 1.6-arcsec-diameter circle, and corrected for Earth-Titan, Sun-Titan, and phase angle effects, following Lockwood et al. (1986a). It is seen that Titan has dimmed in the blue, while it has brightened sharply at $889 \mathrm{~nm}$. The change in albedo over the 3-year period 1994-1997 is broadly the same as for the 1993-1995 period, as measured by Karkoschka (1998) using ground-based CCD spectroscopy. Both those data and our data indicate a slightly larger change than do Lockwood's photometry data, although the $\sim 1 \%$ difference is probably accountable to experimental error.

We believe the jump in brightness in our data at $889 \mathrm{~nm}$ is real. This $7 \%$ increase in brightness suggests that future monitoring of Titan's reflectivity at this wavelength would be well worthwhile. The brightening implies that there is more haze at high altitudes (above the bulk of the methane) in 1997 than in 1994. It should be noted that the changes in the haze need to be taken into account

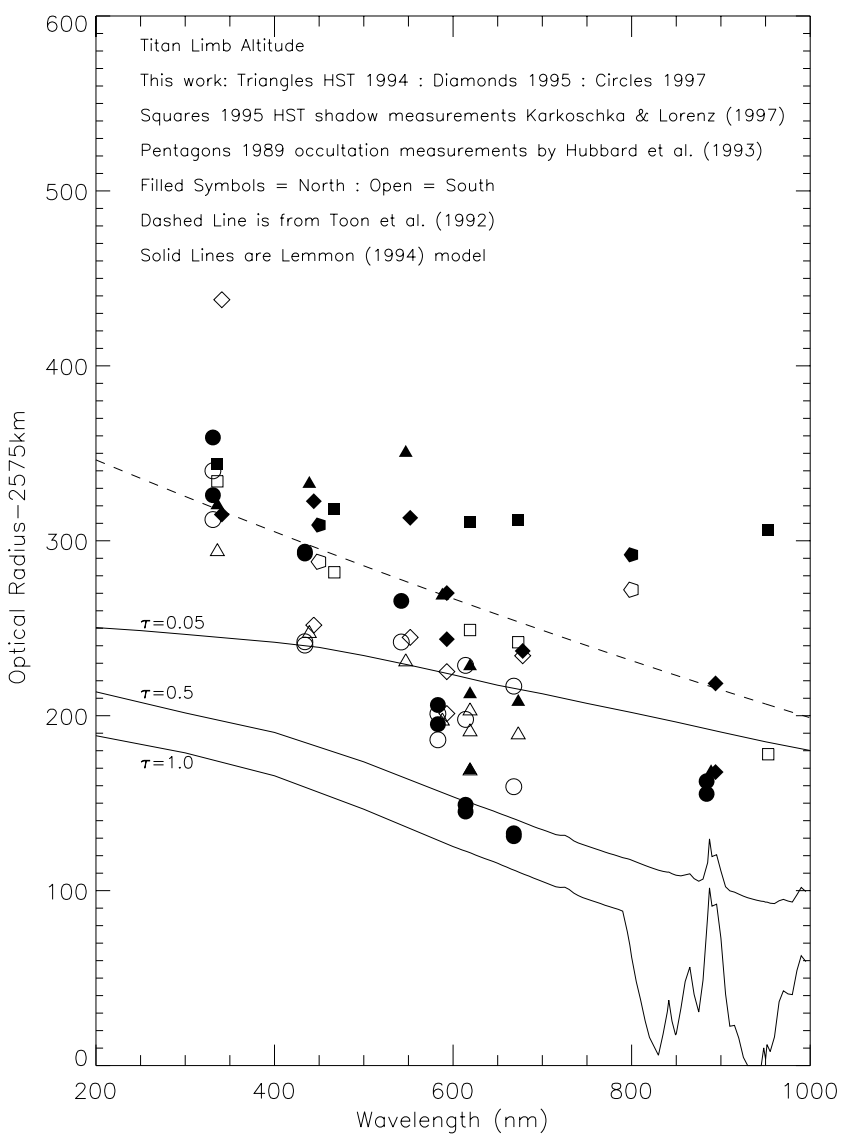

FIG. 5. Model radius as measured from the HST images analyzed in this paper, compared with previous determinations of optical radius. Dashed line shows the analytic expression from Toon et al. (1992), while solid lines show the altitude at which various (vertical) optical depths are attained in the nominal Lemmon (1994) model; note the methane spikes around $889 \mathrm{~nm}$.

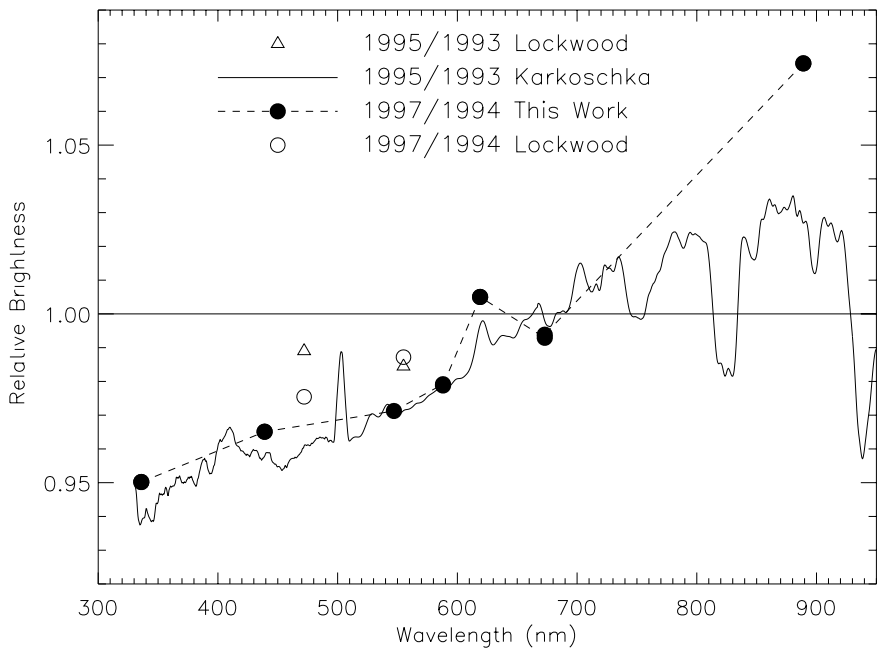

FIG . 6. Variation with time of Titan's brightness. Solid symbols are relative albedo of Titan in our images, corrected for phase angle. Between 1994 and 1997 Titan has become about $5 \%$ fainter in blue, while the red albedo is nearly constant; this same behavior was observed between 1993 and 1995 by Karkoschka (1998). The dramatic ( 7\%) brightening at $889 \mathrm{~nm}$ is significantly higher than for the 1993-1995 period, and suggests more haze at higher altitudes.

in interpreting spectroscopy in terms of surface reflectivity if data spanning several years are to be analyzed, e.g., Coustenis et al. (1995).

\section{MODELS OF SEASONAL CHANGE}

\subsection{Previous Analytic Models}

A sinusoidally varying NSA combines with the varying contribution of north and south hemispheres due to Titan's obliquity (again, roughly sinusoidal, with the same 29.5-year period, but $90^{\circ}$ out of phase) to give a double-frequency signal as observed; see Smith et al. (1981) and especially S81. As noted by Lockwood et al. (1986b) and Sromovsky et al. (1986), the observed NSA was insufficient to produce the observed albedo variation. The discrepancy appeared, over the 19721986 period, to be correlated with the solar cycle. L97 examined these and other models in the light of HST observations in the 1990-1995 period. They found that the combined NSA and albedo data were best matched by a combined sinusoidal NSA and sinusoidal albedo increment to both hemispheres with a 14.5-year period. They suggested that a change in optical radius or Minnaert coefficient, corresponding to a change in haze density at high altitudes, might instead account for the discrepancy. The solar cycle mechanism, consistent with the data until 1986, falls out of phase with the required effect in the 1986-1995 period.

A second difficulty with the simple sinusoidal model is that the real cycle seems slightly asymmetric; a fit to the 1979-1981 data yields too high an amplitude for the 1990-1995 period. This may 
be corrected, perhaps artificially, if the southern hemisphere has a higher underlying brightness than the northern one, although this difference must still be due to atmospheric properties, since no blue light reaches the surface.

A third difficulty with the sinusoidal model is one introduced by the new data in this paper. If the phase of the NSA is tuned to fit the extrema available to L97, the NSA falls more slowly than our observations indicate. A fourth, more philosophical, aspect to these models is that while they are algebraically convenient, and useful in introducing how the effect of a seasonal asymmetry and Titan's varying aspect combine to produce a 14.5-year albedo variation, they shed little light on what is actually going on on Titan. We therefore turn our attention to more physically based models.

\subsection{Mechanisms and Physical Models}

All proposed mechanisms for producing the asymmetry and the albedo change invoke Titan's haze, since the light at most of the relevant wavelengths reaches unity optical depth at altitudes far above the surface. Photochemical production variations with season (Hutzell et al. 1993) are ruled out due to the long residence time of haze in the atmosphere; the time for haze to coalesce and fall to regions where it accumulates a significant optical depth is far longer than the seasonal time scale, so seasonal variations in production are smoothed out. Variation due to a difference in particle size between hemispheres is inconsistent with the invariance of the asymmetry with observation phase angle (Sromovsky and Fry 1989).

The most likely mechanism involves meridional circulation, either directly by transporting haze particles from one hemisphere to another (e.g., Hutzell et al. 1996) or by modifying the particle number density or optical properties by influencing condensation (Courtin et al. 1991) and/or removal by rainout (L97). All these latter effects may play a role, but the changes we have observed are at least conceptually consistent with the haze transport mechanism, which has already been indicated by measurements of Titan's shadow: Karkoschka and Lorenz (1997) determined that the haze north of $5^{\circ} \mathrm{S}$ was dominated by $0.3-\mu \mathrm{m}$ particles in 1995 , while south of that latitude, the haze layer was of $0.1-\mu \mathrm{m}$ particles, at an altitude up to $100 \mathrm{~km}$ lower. This is consistent with upwelling air motions in the northern (summer) hemisphere, the motion expected in a thermally direct Hadley-type circulation.

\subsection{A Simple Conceptual Model}

The haze, which may be crudely represented by tholins generated in the laboratory (Khare et al. 1984), is dark relative to the bright, Rayleigh-scattering atmosphere below at short $(<500-\mathrm{nm})$ wavelengths. For example, McKay et al. (1989) find that the single-scattering albedo of haze particles varies from only 0.45 at $300 \mathrm{~nm}$ to 0.95 at $600 \mathrm{~nm}$. Thus, as a crude model for the behavior at blue wavelengths, let us consider a black haze layer overlying a deep, brighter atmosphere, and assume that the deep atmosphere does not change. Further, we consider a meridional circulation, with air above this deep layer rising over the subsolar point and descending at the poles. This has the gross effect of transporting haze from the summer hemisphere to the winter one.

As haze is transported from the summer hemisphere to the winter one, the short-wavelength albedo of the winter hemisphere drops rapidly; even a small addition of obscuring haze adds to the absorption optical depth and reduces the brightness. The short-wavelength albedo of the spring hemisphere stays low, however; the albedo increment in the summer hemisphere due to a quantity of haze fleeing the subsolar point is less than the albedo decrement it causes in the winter hemisphere. This is a natural consequence of the nonlinear nature of opacity. As an example, if a parcel of obscuring haze with an optical depth $\delta \tau \sim 0.1$ moves from a dark hemisphere with a large extinction optical depth (1.0, for example) to one with a low optical depth (0.1), the brightness of the darker hemisphere increases by $e^{-0.9}-e^{-1.0}$, or only 0.038 , while the brighter hemisphere is darkened by $e^{-0.1}-e^{-0.2}$, or 0.086 .

The model computes the total brightness by adding the timedependent contributions from two hemispheres as in L97. Each one has an albedo of the form $A_{x} \exp \left(-\tau_{x}\right)$, with $A$ an underlying albedo and $\tau$ representing the amount of dark haze lying above it, with subscript $x$ denoting north or south.

An insolation-haze product for each hemisphere is crudely computed as $P_{x}=S \tau_{x} \cos \left(\Lambda-\phi_{x}\right)$, where $S$ is the relative insolation (which varies by some $20 \%$ over Saturn's elliptical orbit), $\tau_{x}$ is the amount of haze in the hemisphere, $\Lambda$ is the subsolar latitude, and $\phi_{x}$ is an arbitrary angle to maximize and minimize the $P_{x}$ function at the appropriate solstices; here I use $\phi_{\mathrm{N}}=30, \phi_{\mathrm{S}}=-30$. This quantity $P_{x}$ represents something like a "haze potential"; the potential in a given hemisphere is higher when there is more haze there, and when the Sun is furthest from the equator into that hemisphere. The amount of haze that moves from one hemisphere to the other is a multiple $k 1$ of the difference between these potentials for the two hemispheres.

Additionally, since haze being lofted by the Hadley cell darkens more effectively than haze lower down (since in reality the dark haze and bright atmosphere are vertically mixed), both hemispheres are further darkened by an amount $e^{-k 2 q}$, where $q$ is the sum of the haze potentials and $k 2$ is a constant.

Results of the model are shown in Fig. 7. The model as described above (shown as the dashed curve) fits the 1994-1997 NSA data very well, although it does not quite match the 19791981 data. The fit to the total brightness is no worse than for a sinusoidal model, but can be improved substantially by shifting the curve to the right by 2.5 years. Both models have initial (1952) values $\tau_{\mathrm{N}}=0.9, \tau_{\mathrm{S}}=0.12, k 1=0.005, k 2=30$, and $A_{\mathrm{S}} / A_{\mathrm{N}}=1.03$, and the time step is 0.05 year. It is emphasized that the $\tau_{\mathrm{N}}$ and $\tau_{\mathrm{S}}$ values refer to the haze above some "optical 

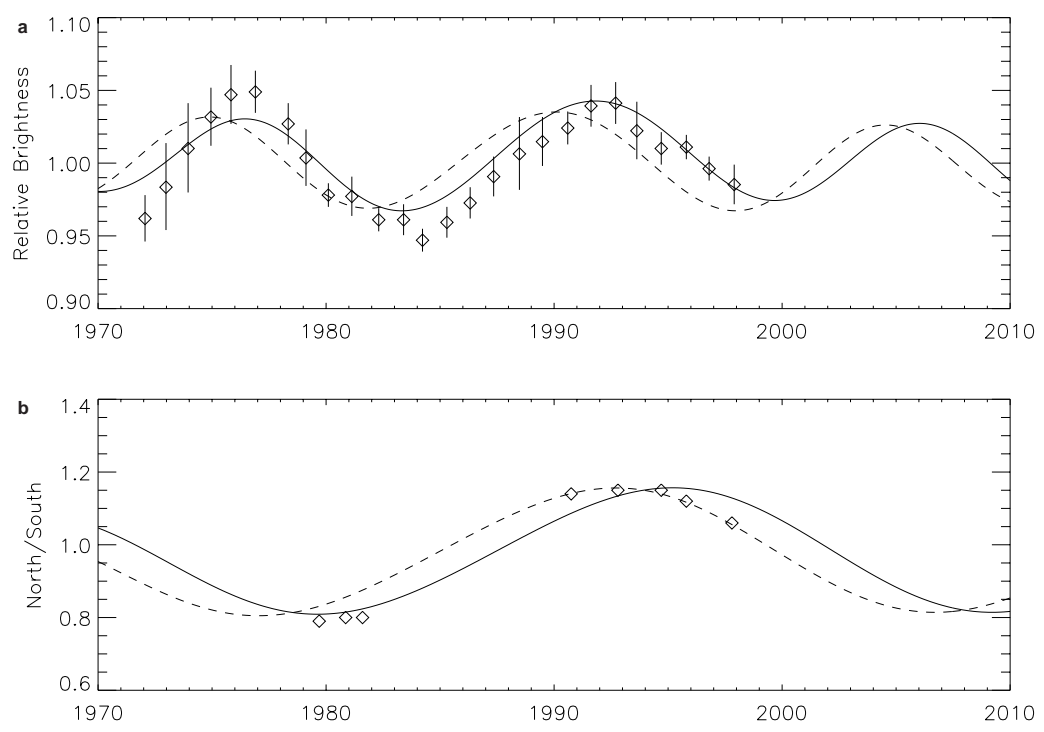

FIG. 7. (a) Disk-averaged reflectivity (i.e., weighted sum of the two hemispheric albedos), with the photometric observations indicated by diamonds with $1 \sigma$ error bars. (b) Ratio of the two model hemispheres, together with Pioneer, Voyager, and HST measurements from L97 and the present paper (error bars are of the order of the symbol size and so are not shown). The dashed curve corresponds to the (preferred) baseline model, while the solid curve (which has a better fit with the brightness data, but somewhat poorer fit for the 1994-1997 HST data) is phase-delayed by 2.5 years.

surface" in the atmosphere, not the optical depth of the atmosphere as a whole.

The rms errors for the "raw" model are 0.024 and 0.039 for the brightness and albedo ratios, respectively, and 0.013 and 0.038 for the "phase-shifted" model. These may be compared with corresponding errors of 0.015 and 0.045 for the sinusoidal model mentioned in the earlier section. It may be noted that phase-shifting the model to fit the brightness curve degrades the 1994-1997 NSA fit, and vice versa. The rapid change in brightness in the early 1970s (around perihelion) is difficult for the models to capture, even though the model takes Saturn's orbital eccentricity into account (so that subsolar latitude varies rapidly and insolation is higher at perihelion).

This simplistic dark-haze model is clearly inappropriate for longer wavelengths where the haze is scattering rather than absorbing and light penetrates deeper into the atmosphere. Fully coupled haze/circulation models may describe the haze transport rather better than the crude formulation above. However, despite its simplicity, this model has a physical basis and does a fair job of reproducing Titan's behavior.

\subsection{Radiative Transfer Modeling}

We now attempt to elucidate the altitudes responsible for the NSA using detailed radiative transfer models.An additional layer of particles with a specified optical depth, single-scattering albedo is inserted into the "baseline" Titan haze microphysics and radiation transfer model (Lemmon 1994) at a specified altitude. The baseline model assumes fractal particles and is tuned to reproduce the Pioneer 11 polarimetry data (Tomasko and Smith 1982) and the ground-based geometric albedo data of Neff et al. (1984). Although this approach is somewhat imperfect-ideally, if there were enough data present to constrain them, two hemisphere models would be made and added together to fit the observed spectrum-it is adequate for the present purpose. A further imperfection, also intractable with the data on hand, is that a variety of altitudes, some perhaps increasing their haze density of one size range of haze particles while simultaneously decreasing density for another size, may be responsible for the albedo change, while to keep the number of free parameters tractable we consider only a single additional layer.

In general, a larger layer optical depth and a higher altitude of insertion increase the effect of the particles. The effect depends on the single-scattering albedo $\omega$. The threshold $\omega$ above which the extra haze brightens Titan rather than darkens it depends on the wavelength. Since all the haze is bright at long wavelengths, a very high $\omega$ is needed to have a brightening effect. These effects are shown in Fig. 8.

To fit our simulated "dark hemisphere" (i.e., the baseline model spectrum, divided by the 1994 NSA) we force the singlescattering albedo of the particles to have values appropriate to tholins, taken from McKay et al. (1989); the range $\omega$ is allowed to take is given in Table I.

We find that the 673-nm filter is not a particularly useful constraint, at least for the single-scattering albedo values used, since viable solutions are present for a large altitude and optical depth range (largely because the NSA is so small at this wavelength). The 336- and 439-nm data argue for a haze layer at 
a

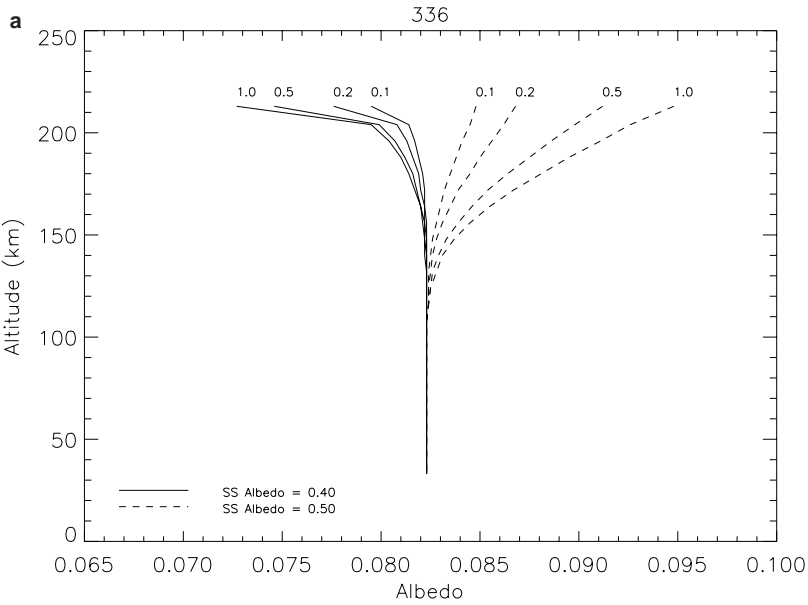

c

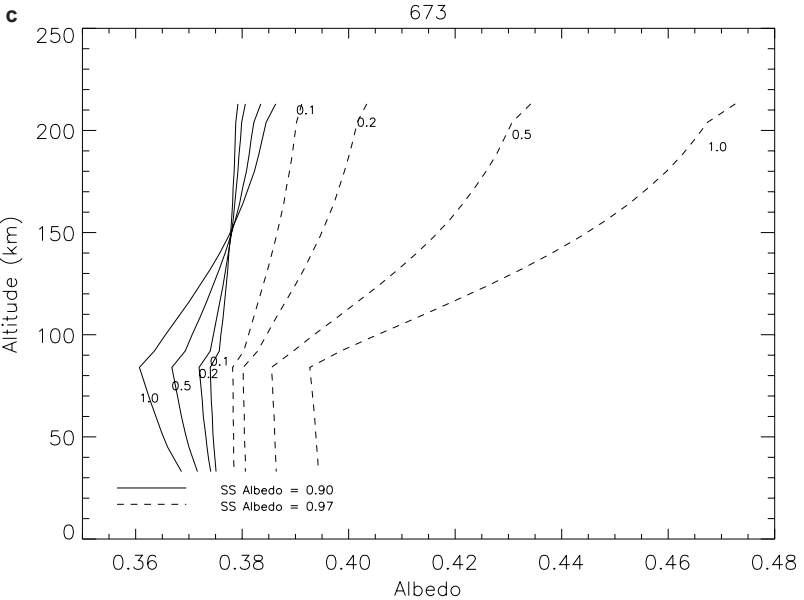

b

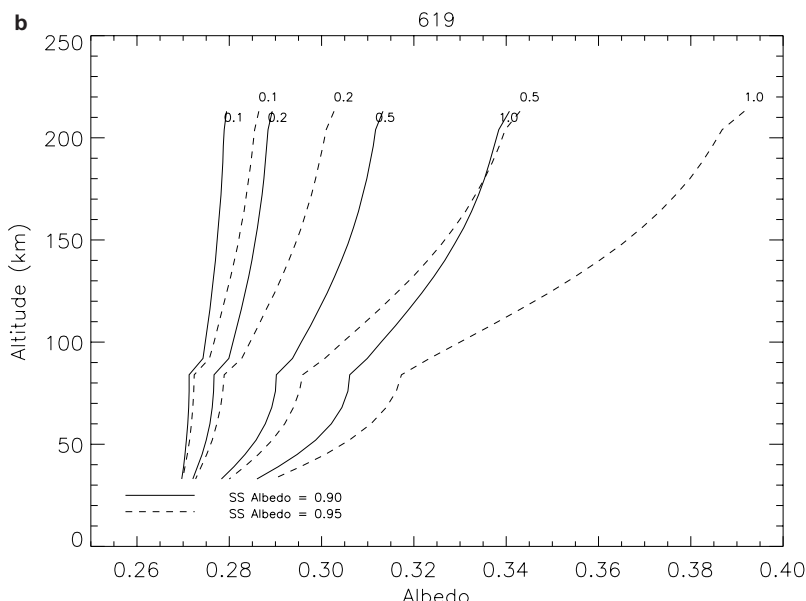

d

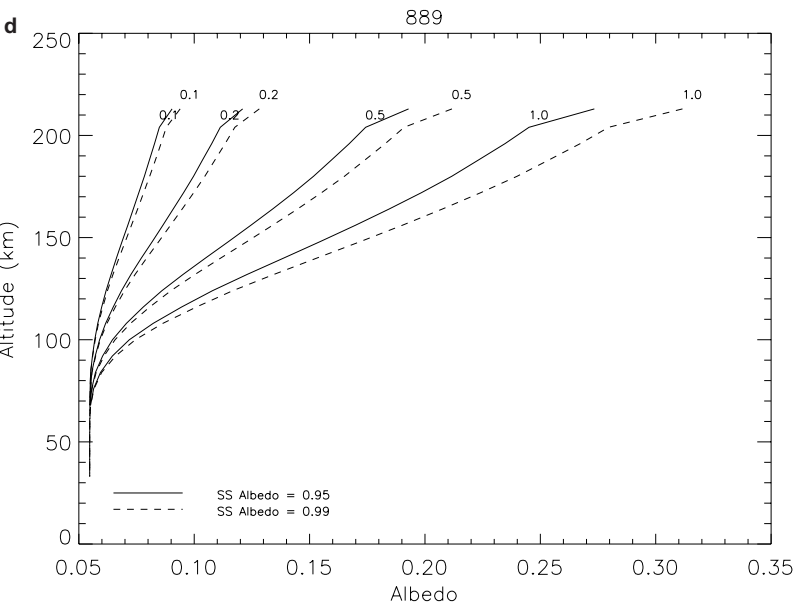

FIG. 8. Radiative transfer model albedo (abscissa) of Titan, with an additional haze layer inserted at an altitude indicated by the ordinate. (a) 336 nm: The effect of the haze is minimal below $100 \mathrm{~km}$ because the "baseline" haze optical depth is high even at that level (see Fig. 5). Above that altitude, bright particles increase the albedo, with thicker layers increasing the albedo even more. Dark particles reduce the albedo. (b) $619 \mathrm{~nm}$ : Here the incremental layer increases the albedo since the underlying atmosphere is dark, and has an effect down to much deeper levels in the atmosphere. The change in slope at about $80 \mathrm{~km}$ is due to the rainout of haze at that altitude. There is a strong slope with altitude due to methane absorption. (c) $673 \mathrm{~nm}$ : This is somewhat similar to the 619 -nm case, except note that below $80 \mathrm{~km}$ the albedo change is relatively insensitive to the altitude of the new layer since methane absorption is weak at this wavelength. Note also that the "background" haze is quite bright; adding haze with a single-scattering albedo of 0.9 can reduce the albedo at low altitudes. (d) 889 nm: Here the effect is similar to that at $336 \mathrm{~nm}$ although this is due to the methane absorption optical depth in the atmosphere, rather than the haze.

\section{TABLE I}

\section{Single Scattering Albedos of the A dditional} Haze Layer

\begin{tabular}{lc}
\hline \multicolumn{1}{c}{ Filter } & Single-scattering albedo \\
\hline F336W & $0.4-0.5$ \\
F439W & $0.45-0.55$ \\
F588N & $0.65-0.75$ \\
FQCH4N-B (619 nm) & $0.9-0.95$ \\
F673N & $0.9-0.97$ \\
FQCH4N-D (889 nm) & $0.94-0.99$ \\
\hline
\end{tabular}

Source. Reprinted, with permission from McKay et al. (1989). high $(>150-\mathrm{km})$ altitude, while the combination of the 588- and 619-nm data requires a layer of optical depth $0.05-0.1$ if the same altitude range is considered. The $889-\mathrm{nm}$ data complement the other constraints quite well, forcing a narrow corridor of possibilities.

The acceptable fits above $160 \mathrm{~km}$ are combined in Fig. 9. Overlain are relative Mie extinction efficiencies for $0.1-$ and 0.3- $\mu$ m-radius particles from Karkoschka and Lorenz (1996). It appears that the 619-nm data argue that the particles must be somewhat larger than $0.1 \mu \mathrm{m}$. If particles are (or at least have the optical properties of spheres of ) $0.3 \mu \mathrm{m}$ or larger, then the layer must be quite thin, with an optical depth $\sim 0.1$ at all wavelengths.

Also shown in that figure is the change in total optical depth at $640 \mathrm{~nm}$ to be expected from meridonal and vertical circulation, 


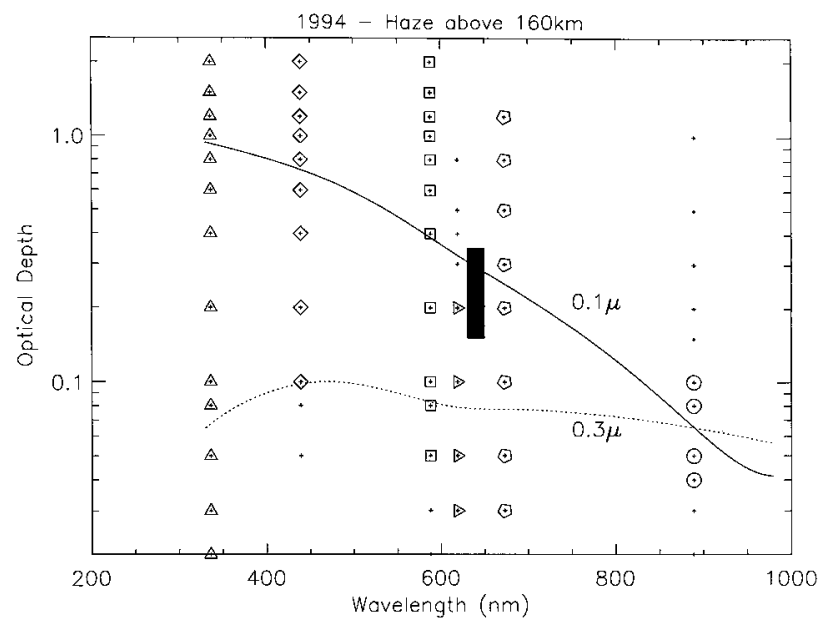

FIG . 9. Assuming a single layer above $160 \mathrm{~km}$ is responsible for the albedo change, the allowable optical depths are shown as a function of wavelength in this figure by symbols. The small crosses indicate parameter sets that were tried but that do not yield the required albedo. The solid line is the optical depth of a layer of $0.1-\mu \mathrm{m}$ radius spherical particles, with the thickness normalized to the $889-\mathrm{nm}$ value, while the dotted line corresponds to $0.3-\mu \mathrm{m}$ radius particles. One-tenth-micron particles seem to be inconsistent with the data at $619 \mathrm{~nm}$, so particles larger than this must be responsible. The shaded box indicates the seasonal change in total optical depth throughout the atmosphere as modeled by Tokano et al. (1999).

as modeled by Tokano et al. (1999) using a coupled GCM/haze model; the change from 0.15 to 0.35 at this wavelength seems compatible with our results, especially since their result is the vertical integral of haze changes throughout the atmosphere, not just above $160 \mathrm{~km}$ as for our result.

The upward vertical winds required to levitate and transport 0.1 - to $1-\mu \mathrm{m}$ particles at these altitudes are only $0.1-1 \mathrm{~cm} / \mathrm{s}$ (Toon et al. 1992), quite compatible with dynamical predictions (Flasar et al. 1981, Hourdin et al. 1995, Tokano et al. 1999). A $1 \mathrm{~cm} / \mathrm{s}$ meridional wind could transport material from $30^{\circ} \mathrm{N}$ to $30^{\circ} \mathrm{S}$ in a few years, compatible with the time scales of the changes we are observing.

\section{CONCLUSIONS}

We have analyzed a suite of images obtained between 1994 and 1997 and have detected significant change in Titan's appearance; the north-south asymmetry has varied more than previous models predicted. The asymmetry over this period appears to be somewhat weaker than in the 1980/1981 epoch.

Until a full cycle of spatially resolved Titan observations has been obtained, any prediction of Titan's seasonal behavior must be tentative. The changes at blue wavelengths are consistent with a simple physical model of upper atmospheric haze transport which we have described. The rapid changes we are observing now are also consistent with the more elaborate model results of Tokano et al. (1999) who show rapid changes in mid- and highlatitude haze optical depth between northern autumn equinox in 1995 and winter solstice in 2002. Both our model and theirs predict that the asymmetry will stay more-or-less fixed for the 2004-2008 duration of the Cassini Mission. While a disappointing prediction, perhaps, this agreement is at least encouraging from a modeling standpoint.

Radiative transfer investigations indicate that the peak (1994) asymmetry is compatible with an additional haze population above $160 \mathrm{~km}$ in the southern hemisphere, with optical depths of $>0.1$ at $439 \mathrm{~nm}$ and $0.04-0.1$ at $889 \mathrm{~nm}$, assuming haze properties equivalent to tholin-like particles $>0.1 \mu \mathrm{m}$ in diameter. Other, more complex, haze variations may be responsible. Additionally, the fractal nature of the aerosol particles (e.g., Rannou et al. 1995, Lemmon 1994) means this radius should be taken only as a guide.

The north-south asymmetry appears to depend both in amplitude and phase on wavelength. We find that changes in violet and yellow/red lead changes in blue. The blue asymmetry may be expected to fall to zero and reverse in the next 1-2 years, while at violet and red wavelengths it has already done so. Titan's behavior at red wavelengths is challenging: it is not clear why changes at these wavelengths should lead changes in blue, nor is it obvious why the model radius of Titan should drop below model estimates at these wavelengths in particular.

We find Titan darker at blue wavelengths by about $5 \%$, while its 889 -nm brightness has increased by some $7 \%$ over the 3 -year period of this study. These changes underscore that Titan is a dynamic object, and it is no longer appropriate to state or use Titan's brightness in models as if it were an unchanging quantity; seasonal changes must be considered.

One useful future investigation will be to compare the observed contrasts of specific surface features as a function of latitude and time with model predictions (i.e., sampling the transmissivity of the haze, rather than its reflectivity). A better model treatment of the haze will entail separate models for each hemisphere; spatially resolved spectroscopy (e.g., using the Space Telescope Imaging Spectrometer) will facilitate this work. Full three-dimensional models of the haze structure, probably using Monte-Carlo methods, will be indispensable tools for grappling with the coupled complications of Titan's atmosphere; radiative effects are driven by the haze structure, which is controlled by the temperature structure and dynamics, which are in turn controlled by radiation. The computational power required for running such models is no longer prohibitively expensive, so we expect substantial progress in this area in the coming years.

We also look forward to future observations of Titan with HST and Cassini. Our experience suggests continuing surprises are in store.

\section{ACKN OWLEDGMENTS}

This work is based in part on observations with the NASA/ESA Space Telescope, and partial support was provided by NASA through Grant PI314159 from the STScI, which is operated by Association of Universities for Research in Astronomy, Inc., under NASA Contract NAS5-2655. G.W.L. acknowledges the support of NASA Planetary Astronomy Programs NAG5-3948 (current) and 
NAGW-4946 (prior). IDL is a trademark of Research Systems, Inc. We thank the referees for useful comments, and Erich Karkoschka for making his data available to us in electronic form.

\section{REFERENCES}

Burrows, C. J. (Ed.) 1994. Hubble Space Telescope Wide Field and Planetary Camera 2 Instrument Handbook. Space Telescope Science Institute, Baltimore.

Caldwell, J. D., P. H. Smith, M. G. Tomasko, and H. Weaver 1992. Titan: Evidence of seasonal change-A comparison of Voyager and Hubble Space Telescope images. Icarus 103, 1-9.

Courtin, R., R. Wagener, C. P. McKay, J. Caldwell, K.-R. Fricke, F. Raulin, and P. Bruston, 1991. UV spectroscopy of Titan's atmosphere, planetary organic chemistry and prebiological synthesis II. Interpretation of new IUE observations in the 220-335 $\mathrm{nm}$ range. Icarus 90, 43-56.

Coustenis, A., E. Lellouch, J. P. Maillard, and C. P. McKay 1995. Titan's surface: Composition and variability from the near-infrared albedo. Icarus 118, 87-104.

Flasar, F. M., R. E. Samuelson, and B. J. Conrath 1981. Titan's atmosphere: Temperature and dynamics. Nature 292, 693-698.

Hourdin, F., O. Talagrand, R. Sadourny, R. Courtin, D. Gautier, and C. P. McKay 1995. Numerical simulation of the general circulation of the atmosphere of Titan. Icarus 117, 358-374.

Hubbard, W. B., and 45 colleagues 1993 . The occultation of 28 Sgr by Titan. Astron. Astrophys. 269, 541-563.

Hutzell, W. T., C. P. McKay, and O. B. Toon 1993. Effects of time-varying haze production on Titan's geometric albedo. Icarus 105, 162-174.

Hutzell, W. T., C. P. McKay, O. B. Toon, and F. Hourdin 1996. Simulations of Titan's brightness by a two-dimensional haze model. Icarus 119, 112-129.

Karkoschka, E. 1995. Spectrophotometry of the jovian planets and Titan at 300- to 1000-nm wavelength: The methane spectrum. Icarus 111, 174-192.

Karkoschka, E. 1998. Methane, ammonia, and temperature measurements of the jovian planets and Titan from CCD-spectrophotometry. Icarus 133, 134-146.

Karkoschka, E., and R. D. Lorenz 1997. Latitudinal variation of aerosol sizes inferred from Titan's shadow. Icarus 125, 369-379.

Khare, B. N., C. Sagan, E. T. Arakawa, F. Suits, T. A. Callcott, and M. W. Williams 1984. Optical constants of organic tholins produced in a simulated titanian atmosphere: From soft X-ray to microwave frequencies. Icarus $\mathbf{6 0}$, 127-137.

Lemmon, M. T. 1994. Properties of Titan's Haze and Surface. Ph.D. thesis, University of Arizona.

Lockwood, G. W., and D. T. Thompson 1979. A relationship between solar activity and planetary albedos. Nature 280, 43-45.

Lockwood, G. W., B. L. Lutz, D. T. Thompson, and E. S. Bus 1986a. The albedo of Titan. Astrophys. J. 303, 511-530.

Lockwood, G. W., D. T. Thompson, and L. A. Sromovsky 1986b. Photometry of Titan: Evidence supporting the seasonal contrast model of albedo variations. Bull. Am. Astron. Soc. 18, 809.
Lorenz, R. D., P. H. Smith, M. T. Lemmon, E. Karkoschka, G. W. Lockwood, and J. Caldwell 1997. Titan's north-south asymmetry from HST and Voyager imaging: Comparisons with models and groundbased photometry. Icarus 127, 173-189.

Neff, J. S., D. C. Humm, J. T. Bergstrahl, A. L. Cochran, W. C. Cochran, E. S. Barker, and R. G. Tull 1984. Absolute spectrophotometry of Titan, Uranus and Neptune: 3500-10500 А Icarus 60, 221-235.

Rages, K., and J. B. Pollack 1980. Titan aerosols: Optical properties and vertical distribution. Icarus 41, 119-130.

Rages, K., and J. B. Pollack 1983. Vertical distribution of scattering hazes in Titan's upper atmosphere. Icarus 55, 50-62.

Rannou, P., M. Cabane, E. Chassefiere, R. Botet, C. P. McKay, and R. Courtin 1995. Titan's geometric albedo: Role of the fractal structure of the aerosols. Icarus 118, 355-372.

Smith, B. A., L. A. Soderblom, R. Batson, P. Bridges, J. Inge, H. Masursky, E. Shoemaker, R. Beebe, J. Boyce, G. Briggs, A. Bunker, S. A. Collins, C. J. Hansen, T. V. Johnson, J. L. Mitchell, R. J. Terrile, M. Carr, A. F. Cook II, J. Cuzzi, J. B. Pollack, G. E. Danielson, A. Ingersoll, M. E. Davies, G. E. Hunt, D. Morrison, T. Owen, C. Sagan, J. Veverka, R. Strom, and V. Suomi 1982. A new look at the Saturn system: The Voyager 2 images. Science 215, 504537.

Smith, B. A., L. A. Soderblom, R. Beebe, J. Boyce, G. Briggs, A. Bunker, S. A. Collins, C. J. Hansen, T. V. Johnson, J. L. Mitchell, R. J. Terrile, M. Carr, A. F. Cook II, J. Cuzzi, J. B. Pollack, G. E. Danielson, A. Ingersoll, M. E. Davies, G. E. Hunt, H. Masursky, E. Shoemaker, D. Morrison, T. Owen, C. Sagan, J. Veverka, R. Strom, and V. Suomi 1981. Encounter with Saturn: Voyager 1 imaging results. Science 212, 163-182.

Smith, P. H. 1980. The radius of Titan from Pioneer Saturn data. J. Geophys. Res. 85, 5943-5947.

Smith, P. H., and M. T. Lemmon 1993. HST images of Titan. Bull. Am. Astron. Soc. 25, 1105. [Abstract]

Smith, P. H., E. Karkoschka, and M. T. Lemmon 1992. Titan's north-south asymmetry from HST images. Bull. Am. Astron. Soc. 24, 950. [Abstract]

Smith, P. H., M. T. Lemmon, R. D. Lorenz, J. J. Caldwell, M. D. Allison, and L. A. Sromovsky 1996. Titan's surface, revealed by HST imaging. Icarus 119, 336-349.

Smith, P. H., R. D. Lorenz, and M. T. Lemmon 1995. New information on Titan's north-south contrast from HST. Bull. Am. Astron. Soc. 27, 1104.

Sromovsky, L. A., V. E. Suomi, J. B. Pollack, R. J. Kraus, S. S. Limaye, T. Owen, H. E. Revercomb, and C. Sagan 1981. Implications of Titan's north-south brightness asymmetry. Nature 292, 698-702.

Tokano, T., F. M. Neubauer, M. Laube, and C. P. McKay 1999. Seasonal variation of Titan's atmospheric structure simulated by a general circulation model. Planet. Space Sci. 47, 493-520.

Tomasko, M. G., and P. H. Smith 1982. Photometry and polarimetry of Titan: Pioneer 11 observations and their implications for aerosol properties. Icarus 51, 65-92.

Toon, O. B., C. P. McKay, C. A. Griffith, and R. P. Turco 1992. A physical model of Titan's aerosols. Icarus $\mathbf{9 5}, 24-53$. 